\title{
Phase Diagram of the Proton-Neutron Interacting Boson Model
}

\author{
J. M. Arias, ${ }^{1}$ J. E. García-Ramos, ${ }^{2}$ and J. Dukelsky ${ }^{3}$ \\ ${ }^{1}$ Departamento de Física Atómica, Molecular y Nuclear, Facultad de Física, Universidad de Sevilla, \\ Apartado 1065, 41080 Sevilla, Spain \\ ${ }^{2}$ Departamento de Física Aplicada, Universidad de Huelva, 21071 Huelva, Spain \\ ${ }^{3}$ Instituto de Estructura de la Materia, CSIC, Serrano 123, 28006 Madrid, Spain \\ (Received 2 June 2004; revised manuscript received 3 September 2004; published 15 November 2004)
}

\begin{abstract}
We study the phase diagram of the proton-neutron interacting boson model with special emphasis on the phase transitions leading to triaxial phases. The existence of a new critical point between spherical and triaxial shapes is reported.
\end{abstract}

DOI: 10.1103/PhysRevLett.93.212501

PACS numbers: 21.60.Fw, 05.70.Fh, 21.10.Re, 64.60.Fr

Quantum phase transitions (QPT) have become a subject of great interest in the study of several quantum many-body systems in condensed matter, quantum optics, ultracold quantum gases, and nuclear physics. QPT are structural changes taking place at zero temperature as a function of a control parameter (for a recent review, see [1]). Examples of control parameters are the magnetic field in spin systems, quantum Hall systems, and ultracold gases close to a Feshbach resonance, or the holedoping in cuprate superconductors.

The atomic nucleus is a finite system composed of $N$ neutrons and $Z$ protons $(Z+N \approx 100)$. Though strictly speaking QPT take place for large systems in the thermodynamic limit, finite nuclei can show the precursors of a phase transition for some particular values of $N$ and $Z$. In these cases, one finds specific patterns in the low energy spectrum revealing the strong quantum fluctuations responsible for the phase transition [2]. Recently the concept of critical point symmetry has been proposed by Iachello and applied to atomic nuclei. First, the transition from spherical to deformed $\gamma$-unstable shapes was studied and the corresponding critical point called $E(5)$ [3]. Since then, the interest in nuclear shape-phase transitions has been constantly growing. The characteristics of the critical point in the phase transition from spherical to axially deformed nuclei, called $X(5)$, were presented in Ref. [4]. More recently, the critical point in the phase transition from axially deformed to triaxial nuclei, called $Y(5)$, has been analyzed [5]. In all these cases, critical points are defined in the context of the collective Bohr Hamiltonian [6]. Using some simplifying approximations, precise parameter-free predictions for several observables are obtained. This allows to identify nuclei at the critical points looking at spectroscopic properties. Indeed, some experimental candidates to critical nuclei have already been proposed [7,8].

The collective Bohr Hamiltonian, underlying this approach to critical point symmetries, is closely related to the interacting boson model (IBM) [9]. The simplest version of the IBM is called IBM-1 since in it no explicit distinction is made between protons and neutrons. In
IBM-1 there are three dynamical symmetries: SU(5), $\mathrm{O}(6)$, and $\mathrm{SU}(3)$. These correspond to well-defined nuclear shapes: spherical, deformed $\gamma$-unstable, and prolate axial deformed, respectively. The structure of the IBM-1 Hamiltonian allows to study systematically the transition from one shape to another. There were some pioneering works along these lines in the 1980s [10-12], but it has been the recent introduction of the concept of critical point symmetry that has recalled the attention of the community to the topic of quantum phase transitions in nuclei. The phase diagram of the IBM-1 has been studied from several points of view [10-15]. The three different phases are separated by lines of first order phase transition, with a singular point in the transition from spherical to deformed $\gamma$-unstable shape that is second order. In the usual IBM-1, no triaxial shapes appear. These can only be stabilized with the inclusion of specific three-body forces. A more natural way to generate triaxial deformations is by explicitly taking into account the proton-neutron degree of freedom with the more realistic IBM-2 [16].

In this Letter we will study the phase diagram of the IBM-2 using a simplified Hamiltonian that keeps all the main ingredients of the most general one. This is the consistent- $Q$ IBM-2 Hamiltonian [17]

$$
H=x\left(n_{d_{\pi}}+n_{d_{\nu}}\right)-\frac{1-x}{N} Q^{\left(\chi_{\pi}, \chi_{\nu}\right)} \cdot Q^{\left(\chi_{\pi}, \chi_{\nu}\right)},
$$

where $n_{d}=\sum_{\mu} d_{\mu}^{\dagger} d_{\mu}, \quad Q^{\left(\chi_{\pi}, \chi_{\nu}\right)}=\left(Q_{\pi}^{\chi_{\pi}}+Q_{\nu}^{\chi_{\nu}}\right) \quad$ with $Q_{\kappa}^{\chi}=\left[d_{\kappa}^{\dagger} \tilde{s}_{\kappa}+s_{\kappa}^{\dagger} \tilde{d}_{\kappa}\right]^{2}+\chi_{\kappa}\left[d_{\kappa}^{\dagger} \tilde{d}_{\kappa}\right]^{2}$, and $N$ is the total number of bosons, which is equal to the number of valence proton plus neutron pairs. The IBM phase diagram studied up to now corresponds to the selection $\chi_{\pi}=$ $\chi_{\nu}$, which produces either spherical, axial, or $\gamma$ independent shapes. We will extend the previous works on IBM phase transitions by exploring the transitions from axial to triaxial shapes within the mean field or intrinsic state formalism. The trial wave function is the most general proton-neutron boson condensate $[18-20], \quad|g\rangle=$ $\left|N_{\pi}, N_{\nu}, \beta_{\pi}, \gamma_{\pi}, \beta_{\nu}, \gamma_{\nu}, \Omega\right\rangle$ 


$$
|g\rangle=\frac{\left(\Gamma_{\pi}^{\dagger}\right)^{N_{\pi}} \hat{R}_{3}(\Omega)\left(\Gamma_{\nu}^{\dagger}\right)^{N_{\nu}}}{\sqrt{N_{\pi} ! N_{\nu} !}}|0\rangle
$$

with

$$
\begin{aligned}
\Gamma_{\kappa}^{\dagger}= & \frac{1}{\sqrt{1+\beta_{\kappa}^{2}}}\left[s_{\kappa}^{\dagger}+\beta_{\kappa} \cos \gamma_{\kappa} d_{\kappa 0}^{\dagger}+\frac{1}{\sqrt{2}} \beta_{\kappa} \sin \gamma_{\kappa}\right. \\
& \left.\times\left(d_{\kappa 2}^{\dagger}+d_{\kappa-2}^{\dagger}\right)\right],
\end{aligned}
$$

where $\kappa=\pi, \nu$ and $\hat{R}_{3}(\Omega)$ is the three-dimensional rotation operator with $\Omega$ fixing the relative orientation (Euler angles) between the proton and neutron condensates. $N_{\pi}$ and $N_{\nu}$ are the numbers of valence proton and neutron pairs, respectively. The equilibrium values of the structure parameters $\left(\beta_{\pi}, \gamma_{\pi}, \beta_{\nu}, \gamma_{\nu}, \Omega\right)$ and the energy of the system for given values of the control parameters in the Hamiltonian $\left(x, \chi_{\pi}, \chi_{\nu}\right)$ can be obtained by minimizing the expectation value of the Hamiltonian (1) in the intrinsic state (2): $\delta\langle g|H| g\rangle=0$. Although there is an explicit dependence of the energy on the Euler angles, it has been shown [20] that oblique configurations (relative orientation angles different from the aligned $\Omega=0$ or the perpendicular $\Omega=\pi / 2$ ) require a repulsive hexadecapole $\pi-\nu$ interaction. Therefore, since our Hamiltonian (1) has no hexadecapole terms, we do not expect oblique configurations. We can then safely assume that any arbitrary local minimum will have $\Omega=0, \gamma_{\pi}=$ $\gamma_{\nu}=0^{\circ}$ (or equal to $60^{\circ}$ ) for the aligned configurations or $\Omega=0, \gamma_{\pi}=0^{\circ}, \gamma_{\nu}=60^{\circ}$ (or $\gamma_{\pi}=60^{\circ}, \gamma_{\nu}=0^{\circ}$ ) for the perpendicular configurations. In both cases, $\Omega=$ 0 , and the rotation operator disappears from the intrinsic state (2). In that situation, the energy per boson in the $\operatorname{limit} N_{\pi}, N_{\nu} \rightarrow \infty$ reduces to

$$
\begin{aligned}
E\left(\beta_{\pi}, \gamma_{\pi}, \beta_{\nu}, \gamma_{\nu} ; \chi_{\pi}, \chi_{\nu}, x\right)= & x \sum_{\kappa=\pi, \nu} \frac{\beta_{\kappa}^{2}}{1+\beta_{\kappa}^{2}}-\frac{1-x}{4} \\
& \times \sum_{\mu=0, \pm 2}\left[\sum_{\kappa=\pi, \nu} Q_{\mu}^{2}(\kappa)\right. \\
& \left.+2 Q_{\mu}(\pi) Q_{-\mu}(\nu)\right],
\end{aligned}
$$

where we have used the notation $Q_{0}(\kappa)=Q_{0}(\beta, \gamma, \chi)_{\kappa}$ and $\quad Q_{2}(\kappa)=Q_{-2}(\kappa)=Q_{2}(\beta, \gamma, \chi)_{\kappa}=Q_{-2}(\beta, \gamma, \chi)_{\kappa}$ with

$$
\begin{aligned}
& Q_{0}(\kappa)=\frac{1}{1+\beta_{\kappa}^{2}}\left[2 \beta_{\kappa} \cos \gamma_{\kappa}-\sqrt{\frac{2}{7}} \beta_{\kappa}^{2} \chi_{\kappa} \cos \left(2 \gamma_{\kappa}\right)\right], \\
& Q_{2}(\kappa)=\frac{1}{1+\beta_{\kappa}^{2}}\left[\sqrt{2} \beta_{\kappa} \sin \gamma_{\kappa}+\sqrt{\frac{1}{7}} \beta_{\kappa}^{2} \chi_{\kappa} \sin \left(2 \gamma_{\kappa}\right)\right] .
\end{aligned}
$$

As a natural extension of the Casten triangle for IBM-1 [17], the geometrical representation of the IBM-2 is a pyramid with the new triaxial dynamical symmetry $\mathrm{SU}^{*}(3)$ [21] in the upper vertex. Figure 1 shows a pictorial representation of the IBM-2 parameter space. Any point in this space is obtained with the following transforma- tion to polar coordinates (see Fig. 1)

$$
\begin{gathered}
\rho=1-x ; \quad \theta=-\frac{\pi}{3} \frac{\chi_{\pi}-\chi_{\nu}}{\sqrt{7}} ; \\
\phi=-\frac{\pi}{3} \frac{\chi_{\pi}+\chi_{\nu}}{\sqrt{7}} .
\end{gathered}
$$

We have explored the IBM-2 parameter space of Hamiltonian (1) and present here a selected set of calculations in order to establish the IBM-2 phase diagram (a more detailed presentation will be given in a forthcoming publication). We have not found traces of phase transitions in the transition from $\mathrm{O}(6)$ to $\mathrm{SU}^{*}(3)$ in a parallel way as the already known transition from $\mathrm{O}(6)$ to $\mathrm{SU}(3)$ in IBM1 . The $\mathrm{O}(6)$ symmetry is in fact very unstable against small perturbations driving the system out of the dynamical symmetry either to axial deformed or to triaxial shapes depending on the interaction. The $\mathrm{O}(6)$ symmetry itself has been proposed as a critical dynamical symmetry [22].

In Fig. 2 we show the transition $\mathrm{SU}(3) \rightarrow \mathrm{SU}^{*}(3)$ through the edge plotted in Fig. 1. Along this line $x=$ 0 and $\chi_{\pi}=-\sqrt{7} / 2$ are fixed. The relevant control parameter is $\chi_{\nu}$ varying from $-\sqrt{7} / 2$ (equal and aligned quadrupole prolate shapes for protons and neutrons) to $\sqrt{7} / 2$ (quadrupole prolate shape for protons and quadrupole oblate shape for neutrons with perpendicular axis of symmetry [21]). In Fig. 2 we present the results for the ground state energy (in arbitrary units) and the shape parameters $\left(\beta_{\nu}, \gamma_{\nu}\right)$. The resulting proton parameters are $\beta_{\pi}=\sqrt{2}$ and $\gamma_{\pi}=0$ for all values of the control parameter $\chi_{\nu}$. In the limit $\chi_{\nu}=-\sqrt{7} / 2$, we recover the results known from IBM-1: $\beta_{\nu}=\sqrt{2}$ and $\gamma_{\nu}=0$. In the opposite limit $\chi_{\nu}=\sqrt{7} / 2$ the results known from Ref. [21] are obtained: $\beta_{\nu}=\sqrt{2}$ and $\gamma_{\nu}=60^{\circ}$. Around $\chi_{\nu}=0.4035$, a clear shape-phase transition is observed, changing the system from axial $\left(\chi_{\nu}<0.4035\right)$ to triaxial

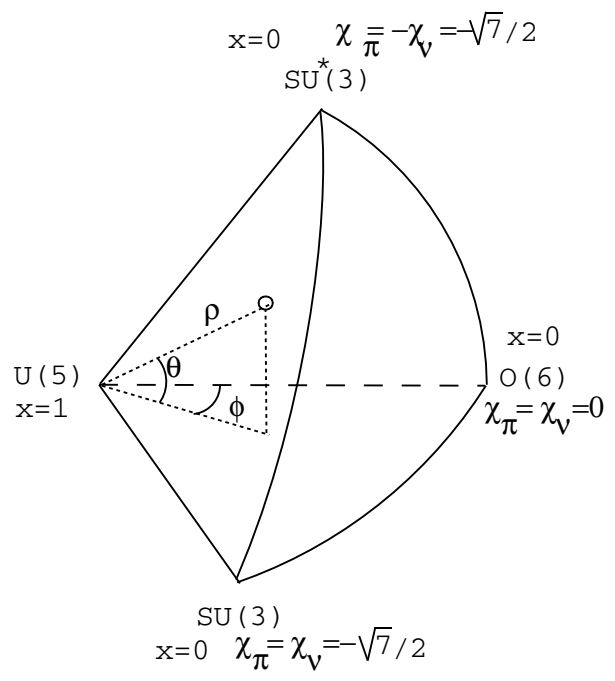

FIG. 1. Pictorial representation of the IBM-2 parameter space with a dynamical symmetry in each of the four vertices. 


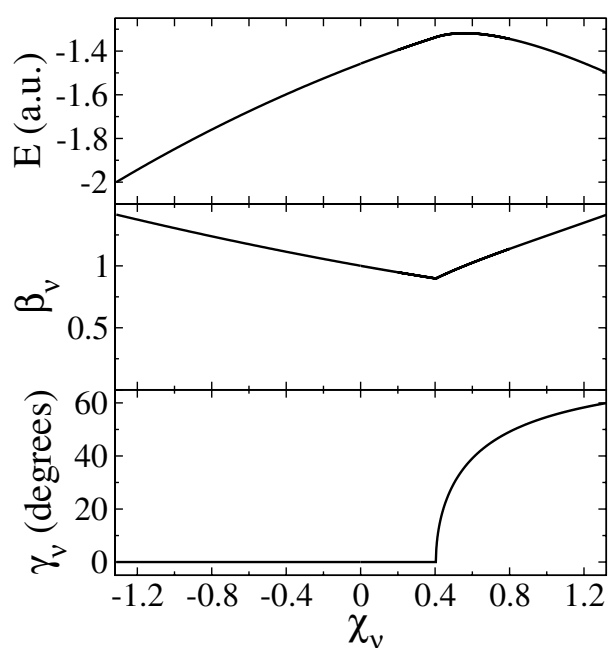

FIG. 2. Transition from $\mathrm{SU}(3)$ to $\mathrm{SU}^{*}(3): x=0, \chi_{\pi}=$ $-\sqrt{7} / 2$, and $\chi_{\nu}$ varies from $-\sqrt{7} / 2$ to $+\sqrt{7} / 2$. In the panels are plotted the energy of the ground state in arbitrary units, and the variation of the shape parameters $\beta_{\nu}$ (dimensionless) and $\gamma_{\nu}$ (degrees).

$\left(\chi_{\nu}>0.4035\right)$. We will call this point " $y$." Note that in this phase transition the order parameter is $\gamma_{\nu}$ that changes from 0 in the symmetric phase to a finite value in the nonsymmetric phase [23]. We have minimized the energy following two inverse paths looking for possible coexistence of minima and the corresponding spinodal and antispinodal points. Both calculations give exactly the same results. This means that spinodal, critical, and antispinodal points all converge to a single point [2]. Therefore, the transition from $\mathrm{SU}(3)$ to $\mathrm{SU}^{*}(3)$ is second order. Figure 3 shows the transition from $\mathrm{U}(5) \rightarrow \mathrm{SU}^{*}(3)$ through the corresponding edge in Fig. 1. Along this edge $\chi_{\pi}=-\chi_{\nu}=-\sqrt{7} / 2$ are fixed and the relevant control parameter is $x$ changing from one (spherical) to 0 (triaxial). The values of $\beta_{\pi}$ and $\beta_{\nu}$ are always equal at the energy minimum, while $\gamma_{\pi}$ and $\gamma_{\nu}$ are symmetric with respect to $\gamma=30^{\circ}$ axis. In the different panels, the energy, and the values of $\beta$ and $\gamma$ for proton and neutron shapes are presented. For $x=1, \beta_{\pi}=\beta_{\nu}=0$, implying a spherical shape. For $x=0$, we recover the $\mathrm{SU}^{*}(3)$ case with $\beta_{\pi}=\beta_{\nu}=\sqrt{2}$, and $\gamma_{\pi}=0$ and $\gamma_{\nu}=60^{\circ}$. A phase transition at $x=0.8$ is observed. We will call this point " $x$." As in the preceding case, we have performed two sets of calculations following inverse paths to determine the order of the transition and again we have found no region of coexistence, converging at the same place, spinodal, critical, and antispinodal points. Note that in this case, the order parameter is $\beta_{\pi}=\beta_{\nu}$, as well as $\gamma_{\pi}=60-\gamma_{\nu}$. In Fig. 4, we present the study of a generic transition from $\mathrm{U}(5)$ (spherical) to a triaxial shape through a trajectory within the IBM-2 pyramid. In particular, we have selected the trajectory defined by $\chi_{\pi}=$ -1.2 and $\chi_{\nu}=0.5$, using $x$ as the control parameter varying from 1 to 0 . The ground state energy, and the 212501-3

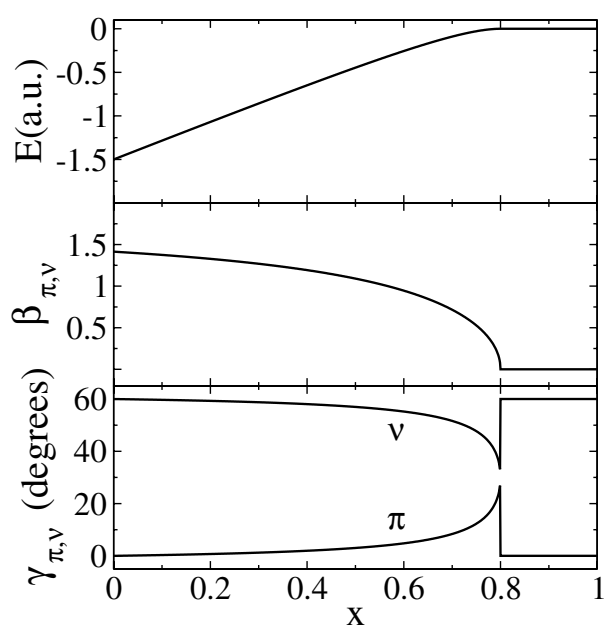

FIG. 3. Same as Fig. 2 but for the transition from $\mathrm{U}(5)$ to $\mathrm{SU}^{*}(3): \chi_{\pi}=-\chi_{\nu}=-\sqrt{7} / 2$ and $x$ varies from 0 (triaxial) to 1 (spherical).

values for the $\beta$ 's and the $\gamma$ 's are plotted. Two phase transitions are observed at different values of $x$. Starting from $x=1$ (spherical system), a first transition to axial deformed shape is observed at $x \approx 0.8$. At this point, the values of $\beta_{\pi}$ and $\beta_{\nu}$ depart from zero but $\gamma_{\pi}$ and $\gamma_{\nu}$ are zero, indicating a deformed axial symmetry. $\beta_{\pi}$ and $\beta_{\nu}$ play the role of order parameters in this phase transition. For a value of $x \approx 0.48$, a second phase transition is observed. The values of $\beta_{\pi}$ and $\beta_{\nu}$ are different from zero in both sides changing smoothly along the transition. The angular parameters $\gamma$ jump from zero to finite values, indicating a transition from an axial shape to a triaxial shape. Therefore, $\gamma_{\pi}$ and $\gamma_{\nu}$ are the order parameters. The different values for the shape parameters

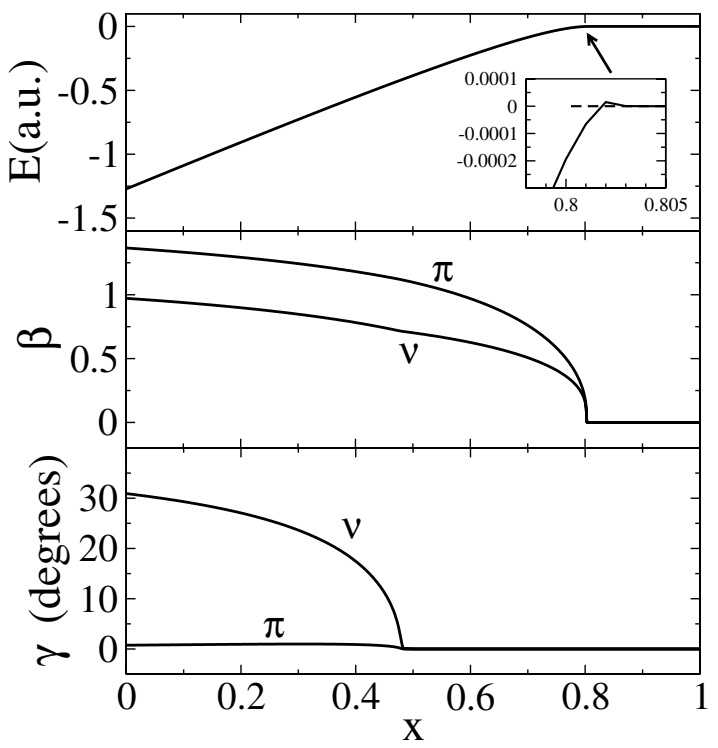

FIG. 4. Same as Fig. 2 but for a generic transition from U(5) to a triaxial shape. The structure parameters are $\chi_{\pi}=-1.2$, $\chi_{\nu}=0.5$, and the control parameter $x$ varies from 0 to 1 .

212501-3 


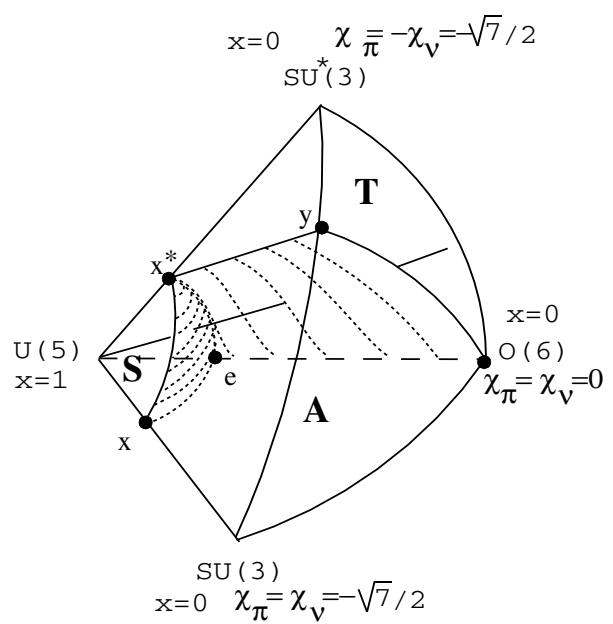

FIG. 5. Schematic phase diagram for IBM-2. S stands for spherical, A for axial, and $\mathrm{T}$ for triaxial phases. The critical points " $x$ " " and " $y$ " studied here and those already known for the IBM-1 phase diagram, " $x$," " $e$," and "O $(6)$ " are marked with dots.

for protons and neutrons are due to the selection of the structure parameters $\chi_{\pi}$ and $\chi_{\nu}$ for this trajectory. As in preceding cases, we have performed two sets of calculations following inverse paths to determine the order of the phase transition. The transition at $x \approx 0.8$ can be analyzed looking at the behavior of the ground state energy (inset). Where the full line corresponds to a forward calculation, starting at $x=0$, increasing $x$, and the dashed line to a backward calculation, starting at $x=1$, decreasing $x$. The inset shows that there are two minima competing, one spherical and one deformed. If the system comes from the spherical region, it keeps spherical for a while even if there is another deformed minimum with slightly lower energy. On the other side, if the system comes from the deformed region, it keeps deformed (look at the small peak in the full line in the inset at $x=0.802$ ) although another spherical minimum has slightly lower energy. This coexistence of deformed and spherical minima in a small region around $x=0.8$ is the signature for a first order phase transition. The phase transition at $x \approx$ 0.48 has been studied with forward and backward calculations. We have not found any coexistence region. The antispinodal, critical, and spinodal points come together to a single point as corresponds to a second order phase transition. We have explored the parameter space of the IBM-2 Hamiltonian (1) in Fig. 1. The resulting phase diagram of the proton-neutron IBM as described by the Hamiltonian (1) is depicted in Fig. 5. There are three well-defined phases: spherical, axially deformed (prolate in the schematic presentation of Fig. 5), and triaxial. The critical surface separating spherical and axially deformed shapes $\left(e-x^{*}-x-e\right)$ is first order, while the surface separating axially deformed and triaxial shapes $\left(e-\mathrm{O}(6)-y-x^{*}-e\right)$ is second order, including the common line between both surfaces $\left(e-x^{*}\right)$. We have checked that in all the cases discussed in which the transition is second order, the behavior of the corresponding order parameter near the critical point is consistent with a critical exponent $1 / 2$ as given by the Landau theory [23]. We would like to stress that the critical surface separating spherical and axially deformed nuclei is almost a sphere with a radius equal to $\rho=0.2$ and centered in $\mathrm{U}(5)$. The straight line plotted inside the figure gives an idea of the trajectory followed by the transition discussed in Fig. 4 . We would like to emphasize that we have found a new critical point $\left(x^{*}\right)$ at the phase transition changing directly from spherical to triaxial shapes. We are currently studying the spectroscopic properties of this critical point. The results will be presented elsewhere. Finally, this scheme of analysis can be easily extended to positive values of $\chi_{\pi}$ to obtain the dynamical symmetry limits $\overline{\mathrm{SU}(3)}$ and $\overline{\mathrm{SU}^{*}(3)}$.

We acknowledge discussions with F. Iachello and $\mathrm{M}$. Caprio. This work was supported in part by the Spanish DGI under Projects No. BFM2002-03315, No. BFM2000-1320-C02-02, No. BFM2003-05316C02-02, and No. FPA2003-05958.

[1] M. Vojta, Rep. Prog. Phys. 66, 2069 (2003).

[2] F. Iachello and N.V. Zamfir, Phys. Rev. Lett. 92, 212501 (2004).

[3] F. Iachello, Phys. Rev. Lett. 85, 3580 (2000).

[4] F. Iachello, Phys. Rev. Lett. 87, 052502 (2001).

[5] F. Iachello, Phys. Rev. Lett. 91, 132502 (2003).

[6] A. Bohr and B. Mottelson, Nuclear Structure (Benjamin, New York, 1975), Vol. II.

[7] R. F. Casten and N.V. Zamfir, Phys. Rev. Lett. 85, 3584 (2000).

[8] R. F. Casten and N. V. Zamfir, Phys. Rev. Lett. 87, 052503 (2001).

[9] F. Iachello and A. Arima, The Interacting Boson Model (Cambridge University Press, Cambridge, England, 1987).

[10] A. E. L. Dieperink et al., Phys. Rev. Lett. 44, 1747 (1980).

[11] D. H. Feng et al., Phys. Rev. C 23, 1254 (1981).

[12] A. Frank, Phys. Rev. C 39, 652 (1989).

[13] E. López-Moreno and O. Castaños, Phys. Rev. C 54, 2374 (1996).

[14] J. Jolie et al., Phys. Rev. Lett. 89, 182502 (2002).

[15] J. M. Arias et al., Phys. Rev. Lett. 91, 162502 (2003).

[16] A. Arima et al., Phys. Lett. B 66, 205 (1977).

[17] R. F. Casten and D. D. Warner, Rev. Mod. Phys. 60, 389 (1988).

[18] J. N. Ginocchio and M.W. Kirson, Nucl. Phys. A350, 31 (1980).

[19] A. Bohr and B. Mottelson, Phys. Scr. 22, 468 (1980).

[20] J. N. Ginocchio and A. Leviatan, Ann. Phys. (N.Y.) 216, 152 (1992).

[21] A. E. L. Dieperink and R. Bijker Phys. Lett. 116B, 77 (1982).

[22] J. Jolie et al., Phys. Rev. Lett. 87, 162501 (2001).

[23] J. J. Binney, N. J. Dowrick, A. J. Fisher, and M. E. J. Newman, The Theory of Critical Phenomena (Clarendon Press, Oxford, 1992). 